\title{
Die Fehlrezeption von Max Webers Studie über „Hinduismus und Buddhismus “ in Indien: Ursachen und Folgen*)
}

\section{Detlef Kantowsky}

Universität Konstanz, Fachgruppe Soziologie, Postfach 5560, D-7750 Konstanz

Zusammenfa ssung: Max Webers Studie über „Hinduismus und Buddhismus“ wurde in Indien (und sicher nicht nur dort) aus mindestens den folgenden vier Gründen mißverstanden. Zum einen ist der Kontext und das argumentative Umfeld der Studie in Indien kaum bekannt; zum zweiten liegt sie nur in einem Übersetzungsentwurf vor; drittens wurde sie im Sinne eines evolutiven Geschichtsverständnisses über die USA „parsonifiziert" vermittelt; und viertens schließlich will das Glück auch der indischen, von den Grundlagen der eigenen Kultur entfremdeten sozialwissenschaftlichen Intelligenz legitim sein.

Max Webers Studie über „Hinduismus und Buddhismus" (Weber 1921) wurde in Indien aus mindestens den folgenden vier Gründen mißverstanden:

- einmal ist der Kontext und das argumentative Umfeld in Indien kaum bekannt;

- zum zweiten liegt sie nur in einem Übersetzungsentwurf vor;

- drittens wurde sie „parsonifiziert“ vermittelt;

- viertens schließlich will das Glück sozialwissenschaftlicher Intelligenz auch in Indien legitim sein.

Das indische Quellenmaterial und weitere Details über das regional-spezifische Umfeld der Argumentation sind in zwei 1982 erschienenen Arbeiten nachzulesen (Kantowsky 1982a und 1982b), bei deren Fertigstellung aber "The Monologue“ von Don Martindale (Martindale 1982) leider noch nicht vorlag - ein Text, der meine schlimmsten Befürchtungen hinsichtlich der Werkgeschichte der Übersetzung von „Hinduismus und Buddhismus" noch weit übertrifft. Insofern begrüße ich die Gelegenheit, hier noch einmal pointierter darstellen zu können, was mich bei meinen Arbeiten über die Rezeptionsgeschichte von "Hinduismus und Buddhismus" beschäftigt hat.

Eine erläuternde Einleitungsbemerkung vielleicht noch vorweg für diejenigen, die fragen mögen, wo jenseits des akademischen Interesses an sich selbst die Bedeutung der Fragestellung denn wohl liegen könnte.

* Geringfügig ergänzte Fassung meines Beitrags zur Tagung „Die Rationalisierungsthese Max Webers in Verhältnis zum Hinduismus und zum Buddhismus“. Universität Turin/Goethe Institut Turin, 24./25. 11. 1983.
Ich bin sicher, daß Pietro Rossi eine vorzügliche Übersetzung der „Gesammelten Aufsätze zur Religionssoziologie" seinen italienischen Lesern vorgelegt hat (Weber 1982), auch wenn ich das selbst wegen mangelnder Sprachkenntnisse leider nicht nachprüfen kann. Es ist aber in diesem Fall wirklich ein eher akademisches Problem, wie gut oder mangelhaft die Übersetzung ist, denn in venezianische Spiegel schauen indische Zeitgenossen ja eher selten. Viktorianische Spiegel dagegen, englische Texte also, können von eminenter Bedeutung sein, weil sie von Indern eingesehen werden können. Wie ein Inder also das Bild seiner eigenen Gesellschaft wahrnimmt, das wir in der gegenwärtig dominanten Industriekultur reflektieren (Ernst/Kantowsky 1983), kann - es muß nicht! - Ausgangspunkt für sozio-kulturelle Neubesinnung und Abgrenzung (vgl. die Wirkung von Max Mueller) oder die Definition von Unterentwicklung und $\mathrm{Ab}$ hängigkeit sein. Und genau in diesem zweiten Sinn wurde Max Weber bislang in Indien (nur dort?) vermittelt und leider auch rezipiert. Ich verstehe meinen Beitrag daher als einen Versuch, Max Weber vom amerikanischen Kopf wieder auf die deutschen Füße zu stellen, um klarzumachen, daß er das gerade nicht war, wofür er (nicht nur) in Indien ausgegeben wurde, nämlich ein Entwicklungstheoretiker mit dem Anspruch, die okzidentale Form der Welterklärung und -bearbeitung global begründen zu wollen.

Wäre das Wort nicht so inflatorisch vernutzt, würde ich überdies behaupten, daß die Ent-Parsonifizierung Max Webers einen Paradigmen-Wechsel in der deutschen Sozialwissenschaft einleiten und sie aus dem wohlfeilen Gehäuse instrumenteller Vernunft und funktionaler Hörigkeit befreien könnte, das die Verhandlungen auf dem letztjährigen Soziologentag in Dortmund so lähmend-deutlich vorgestellt haben. 


\section{Zum Kontext von ,Hinduismus und Buddhismus"}

1904 bzw. 1905 erscheint der Aufsatz über „Die protestantische Ethik und der Geist des Kapitalismus" im 20. bzw. 21. Band des „Archiv für Sozialwissenschaft und Sozialpolitik“, eine Zeitschrift, die Weber zusammen mit seinen Freunden Werner Sombart und Edgar Jaffé seit Frühjahr 1904 editorisch verantwortet.

Die darin entwickelte These über den Zusammenhang einer spezifisch abendländischen Form innerweltlicher Askese und ihre Auswirkungen in Form „innerweltlichen Handelns“ führt logischerweise zu „Kontraststudien“ der anderen Hochreligionen der Menschheit. Die Arbeiten daran beginnen wohl um 1906, sie werden bei Ausbruch des Ersten Weltkrieges abgebrochen. Dennoch erscheinen seit 1915 die entsprechenden Studien über „Konfuzianismus und Taoismus“, „Hinduismus und Buddhismus“ und „Das antike Judentum“ im „Archiv" unter dem Obertitel: „Die Wirtschaftsethik der Weltreligionen - religionssoziologische Skizzen“.

Im ersten Satz einer erklärenden Fußnote heißt es dazu erläuternd: „Die nachstehenden Darlegungen erscheinen unverändert so wie sie vor zwei Jahren niedergeschrieben und Freunden vorgelesen waren. Einziehung zum Dienst machte es unmöglich, den wissenschaftlichen ,Apparat', wie beabsichtigt, beizufügen; an seiner Stelle sind kurze Hinweise auf die Literatur bei Beginn jedes Abschnittes beigegeben." (Archiv, Vol. 41, 1915, S. 1)

Die Aufsatzreihe wird durch eine „Einleitung“ auf den Seiten 1-30 von Band 41 des „Archivs“ begründet, bevor der Artikel „Der Konfuzianismus I. II." daran anschließend beginnt. In Heft 2 des 41. Bandes des „Archivs“ erscheint dann „Der Konfuzianismus III. IV" und daran anschließend auf S. 387-420 eine „Zwischenbetrachtung. Stufen und Richtungen der religiösen Weltablehnung“.

In Heft 3 des 41. Bandes erscheint: „Die Wirtschaftsethik der Weltreligionen. (Dritter Artikel) Hinduismus und Buddhismus". Die Zwischenbetrachtung hatte im vorhergehenden Heft schon auf den nächsten Aufsatz vorbereitet, wenn es im ersten Satz hieß: „Das Gebiet der indischen Religiosität, in welches wir eintreten wollen, ist im stärksten Kontrast gegen China die Wiege der theoretisch und praktisch weltverneinendsten Formen von religiöser Ethik, welche die Erde hervorgebracht hat."
Die beiden weiteren Folgen der Arbeit über „Hinduismus und Buddhismus" erscheinen dann, jeweils mit „(Fortsetzung)“ bzw. „Schluß“ besonders, anknüpfend gekennzeichnet in Heft 2 (Vol. 42), 1916 und Heft 3 (Vol. 42), 1917.

Die mehrteilige Folge über „Das antike Judentum" erscheint ab Heft 1 (Oktober 1917) von Band 44. Hier finden wir in der erläuternden Fußnote auf Seite 52 erstmals einen öffentlichen Hinweis auf den inzwischen offenbar gereiften Plan, die Studien über die „Wirtschaftsethik der Weltreligionen“ mit denen über die „Protestantische Ethik" in einer eigenen Veröffentlichung zusammenzufassen: „Bei einer künftigen Sammlung und umgearbeiteten (und für China mit Quellenzitaten versehenen und ergänzten) Veröffentlichung dieser in Verbindung mit anderen älteren und einigen noch unpublizierten Aufsätzen wird der fehlende Teil eingefügt werden.“

Damit sind wir also bei den drei Bänden der „Gesammelten Aufsätze zur Religionssoziologie“" (GAzRS). Band 1 enthält die überarbeitete und ergänzte Fassung des Aufsatzes über die „Protestantische Ethik" zusammen mit den "Sektenaufsätzen". Er enthält außerdem die ganz wesentlich ausgeweiteten Studien über China, die jetzt 250 statt 108 Seiten lang sind.

Wesentlich überarbeitet ist auch die „Einleitung“ in „Die Wirtschaftsethik der Weltreligionen“, die in der Unter-Überschrift jetzt nicht mehr als ,Religionssoziologische Skizzen“, sondern als „Vergleichende religionssoziologische Versuche" beschrieben werden.

Wesentlich überarbeitet und um längere Passagen ergänzt ist dann schließlich auch die „Zwischenbetrachtung" am Ende des ersten Bandes der GAzRS. Entsprechend bezeichnet Weber sie jetzt auch als „Theorie der Stufen und Richtungen religiöser Weltablehnung".

Eingeleitet wird Band 1 der GAzRS durch eine „Vorbemerkung“ auf den S. 1-16, in der das Projekt der Gesammelten Aufsätze zusammenfassend begründet und theoretisch eingeordnet wird. Diese Vorbemerkung wurde wahrscheinlich im Winter 1919/20 geschrieben. Wir haben darin, sowie in den überarbeiteten Fassungen von „Einleitung“ und "Zwischenbetrachtung" die letzten Stücke theoretischer Reflexion Max Webers.

Band 1 der Gesammelten Aufsätze erscheint am 4. November 1920. Max Weber widmete ihn seiner Frau, wie das wenige Tage vor seinem Tod mit 
Else Jaffé am 7. Juni 1920 auf dem Krankenlager abgesprochen worden war.

Band 2 der GAzRS erscheint am 6. Januar 1921; er ist „Mina Tobler zugeeignet", wie ebenfalls mit Else Jaffé am 7. Juni 1920 besprochen. Er enthält nur die drei Artikel über "Hinduismus und Buddhismus" und ist statt 374 jetzt 378 Seiten lang, weil Weber fünf Ergänzungen eingearbeitet hatte. (Kantowsky 1982b: 161-163)

Ich vertrete die Auffassung, daß Weber nicht mehr noch daran gearbeitet hätte, auch wenn er noch länger hätte leben dürfen. Er wußte, was er wissen mußte, um seine Theorie der unterschiedlichen soziokulturellen Ausprägungen bei der Rationalisierung des Theodizee-Problems $\mathrm{zu}$ begründen. Marianne Weber schreibt allerdings in ihrem „Vorwort zum dritten Band“, der am 10. Februar 1921 ausgeliefert wurde: „Der Verfasser hat die im ersten Band dieser Schriften zusammengefaßten Aufsätze noch überarbeitet und namentlich die Abhandlung über die chinesischen Religionsformen erheblich ergänzen können. Die im zweiten und dritten Band enthaltenen Schriften sind dagegen fast unverändert wie in der ersten Fassung geblieben."

Dieser Deutung Marianne Webers, die damit das Werk ihres Mannes gegen etwaige Angriffe zu immunisieren versuchte, steht der letzte Satz von Fußnote 1 zur Einleitung in Band 1 von GAzRS entgegen (S. 237), die im übrigen die gleiche Entstehungsgeschichte wie schon bei Beginn der Serie im "Archiv" gibt, bloß wurde sie in die Vergangenheitsform gesetzt: „Ich habe bei der jetzigen gesammelten Herausgabe gesucht, neben der Beseitigung einiger kleinerer Versehen auch die starken Unvollkommenheiten der Darstellung, namentlich der chinesischen Verhältnisse, soweit zu bessern, als es dem Nichtfachmann nach Lage des ihm zugänglichen Materials möglich ist und die Quellenzitate etwas vervollständigt."

Soviel zur Werkgeschichte; sie ist wichtig auch und gerade für ein angemessenes Verständnis von „Hinduismus und Buddhismus“, weil diese Studie nur im Zusammenhang - als eine Kontrastanalyse im Sinne Webers richtig aufgefaßt werden kann. Es wäre ihm nie eingefallen, seine Arbeit als eine Monographie über die Religion Indiens auszugeben oder gar als eine Soziologie des Hinduismus und Buddhismus zu bezeichnen, wie es dann in der amerikanischen Übersetzung geschah.

Damit sind wir bei der Übermittlungsgeschichte. Wo kann man die einzelnen, „Hinduismus und
Buddhismus“ mit dem Gesamtwerk der GAzRS verknüpfenden Teilstücke ab wann auf englisch finden?

Die „Vorbemerkung“ übersetzte Talcott Parsons als „Authors's Introduction“. Sie ist seit 1930 in seiner Übersetzung des Aufsatzes über die protestantische Ethik nachzulesen. Im Vorwort des Übersetzers (,Translator's Preface“) bemerkt Parsons erläuternd:

„The Introduction, which is placed before the main essay, was written by Weber in 1920 for the whole series on the Sociology of Religion. It has been included in this translation because it gives some of the general background of ideas and problems into which Weber himself meant this particular study to fit" (Weber 1930: ix-x).

Wie dieses Zitat andeutet, war sich Parsons über die besondere Bedeutung der "Vorbemerkung" schon $1930 \mathrm{im}$ klaren. „Einleitung" und „Zwischenbetrachtung" jedoch schätzte er nicht ihrer Bedeutung gemäß richtig ein, ja er erwähnt die Zwischenbetrachtung gar nicht, sondern schreibt:

„Besides the parts translated, it (i. e. the first volume) contains a short, closely related study, „Die protestantischen Sekten und der Geist des Kapitalismus"; a general introduction to the further studies of particular religions which as a whole he called „Die Wirtschaftsethik der Weltreligionen"; and a long study of Confucianism and Taoism" (Weber 1930: x).

Die „Einleitung“ kann ein sorgfältiger Leser in der Aufsatzsammlung „From Max Weber“ finden, die Hans H. Gerth und C. Wright Mills unmittelbar nach dem zweiten Weltkrieg herausbrachten. Sie gaben ihr den selbsterfundenen Titel: „The Social Psychology of the World Religions" (Gerth/Mills, 1948, 267-301); ihre erläuternde Fußnote gibt fast jedes Detail der Werkgeschichte falsch wieder (Kantowsky 1982b, 145).

Die ,Zwischenbetrachtung" findet man ebenfalls im gleichen Band (Gerth/Mills 1948: 323-59) unter dem Titel: „Religious Rejections of the World and their Directions“. Auch hier wird in der erläuternden Fußnote die Werkgeschichte wiederum falsch dargestellt (Kantowsky 1982b: 146/48); vor allem aber ist den Übersetzern nicht aufgefallen, daß Weber die Überschrift in der zweiten Fassung der „Zwischenbetrachtung“ durch das wichtige Wort "Theorie" ergänzte.

Worauf ist dieser „desolate Zustand“ in der Vermittlung von zwei zentralen Teilstücken des argumentativen Umfeldes von „Hinduismus und Buddhismus" zurückzuführen? Was ist die Vorgeschichte? 
Mit Hilfe seiner Verlobten Hedwig Ide von Reventlow flieht Hans H. Gerth 1938 aus Deutschland über Dänemark nach USA. Er wird 1940 Assistant Professor an der University of Wisconsin, Madison Sociological Department. 1947 wird er, unter anderem wegen des Erfolges der Aufsatzsammlung „From Max Weber“ Full Professor. Wie ist die Übersetzung entstanden?

In seinen Kursen hatte Hans H. Gerth Übersetzungen Weberscher Texte verteilt, die er mit Hilfe seiner Sekretärin hergestellt hatte. Einer seiner Studenten war C. Wright Mills, der sie sammelte und - zunächst ohne Wissen von Hans H. Gerth ein Angebot von Oxford University Press einholte. Er berichtet dann Hans H. Gerth, daß OUP das Buch nur herausbringen wolle, wenn auch sein (Mills) Name mit als Herausgeber erscheine. Gerth stimmt zu und Mills überarbeitet dann die Übersetzungen, während Hans H. Gerth den einleitenden Essay „Max Weber: The Man and His Work" schreibt. Das Gesamtprojekt ist dann einen Moment lang gefährdet, weil Gerth nicht möchte, daß Mills als Mitautor der Einleitung zeichnet (Martindale 1982: 161). Daß man sich im übrigen bei diesem Unternehmen um so etwas wie „Copyrights" nicht sonderlich kümmerte, hat mir Herr Georg Siebeck mit Schreiben vom 2. 12. 1980 mitgeteilt:

Für eine Ausgabe von Gerth/Mills ist es mir nach langwierigen Bemühungen gelungen, ab 1967 - dem Jahr, da mir die Existenz des Bandes ,Essays in Sociology' zur Kenntnis kam - ein Honorar zu bekommen, und zwar $1 \%$ der in Amerika verkauften Exemplare, $10 \%$ von Einnahmen aus Nebenrechten. Aus den selbst bei diesem niedrigen Prozentsatz noch immer beachtlichen Beträgen geht hervor, welche Verluste mir entstanden sind.

Halten wir fest: Das argumentative Umfeld $\mathrm{zu}$ „Hinduismus und Buddhismus“ ist einem englischsprachigen Leser zwar seit 1930 (Vorbemerkung) bzw. 1948 (Einleitung und Zwischenbetrachtung) zugänglich. Er muß aber genau wissen, wo er was warum nachzusehen hat. Hinweise dazu findet er in der Übersetzung von „Hinduismus und Buddhismus" überhaupt nicht. Damit kommen wir zur Übersetzungsgeschichte.

\section{Zur Übersetzung von „Hinduismus und Buddhismus"}

1958 erscheint die Studie unter dem Titel „The Religion of India. The Sociology of Hinduism and Buddhism" bei der Free Press. Bemerkungen zum Kontext, Angaben über die Quelle und zum Origi- nal, Ausführungen über die Stellung der Studie im Rahmen der „Gesammelten Aufsätze zur Religionssoziologie“, einen Hinweis auf die Erstveröffentlichung als einer ,religionssoziologischen Skiz$\mathrm{ze}^{\text {" }}$ über „Die Wirtschaftsethik der Weltreligionen", eine Zusammenfassung der Hauptargumente aus „Vorbemerkung“, „Einleitung“" und „Zwischenbetrachtung“ - all dies sucht der Leser vergeblich. Die englischsprachige Fassung eines Teilstückes einer vergleichenden Aufsatzsammlung mit übergeordneter Fragestellung fällt 1958 quasi als Erstveröffentlichung aus dem nordamerikanischen Verlegerhimmel. (Weber 1958)

Diese auffallende Anonymität ist wohl vor allem darauf zurückzuführen, daß auch diese Übersetzung nicht lizensiert war. Georg Siebeck teilte mir dazu mit Schreiben vom 22. 10. $1980 \mathrm{mit}$ :

„Mit Ihrer Frage, wie weit die Herausgeber und Übersetzer freie Hand hatten, sind Sie in ein wahres Wespennest gestochen: die Übersetzung war überhaupt nicht von uns lizensiert und ist es bislang immer noch nicht. Über diese und andere Ausgaben streiten wir uns seit langer Zeit mit Free Press herum. Nach dem Kriege war durch das Besatzungsrecht so etwas möglich, nämlich daß deutsche Urheberrechte und Patente schlichtweg "geplündert" werden konnten. Die Verluste für die Weber-Familie und natürlich auch den Original-Verlag sind nicht unerheblich, zumal nur in einzelnen Fällen nach äußerst zähen Verhandlungen nachträgliche Vereinbarungen getroffen werden konnten. Noch gravierender ist freilich wie Sie sehr klar nachgewiesen haben - daß die Übersetzungen dadurch völlig unkontrolliert herauskommen konnten."

Leider ist das freibeuterische verlegerische Interesse der „Free Press" aber nur ein Teil der Hintergrundgeschichte. Auf Grund meiner Recherchen in Form von Briefwechseln mit Don Martindale, Nobuko Gerth, Georg Siebeck, Guenther Roth, Johannes Winckelmann, Edward Shils und Irving L. Horrowitz hatte ich schon 1981 starke Zweifel an der "translation“ angemeldet (Kantowsky 1981). Einige Kollegen fanden damals meine Einschätzung dieser Arbeit als „transgression“ etwas zu hart. Inzwischen aber hat Don Martindale seine Geschichte erzählt - wobei es interessant ist, daß sie bei der „Intercontinental Press“, im kleinen Städtchen Ghaziabad in Uttar Pradesh (Indien) beheimatet, verlegt wurde in einer Schriftenreihe, die sich "Intercontinental Series in Sociology“ nennt, deren „Advisory Editors“ Don Martindale und Joseph S. Roucek sind.

Ich empfehle diese Studie jedem, nicht nur als Hintergrundinformation zur "transgression“, sondern vor allem auch als Nachschlagewerk zum 
Thema „Games People play in American Universities". "The Masters" von C. P. Snow liest sich dagegen wie ein idyllischer Roman aus der guten alten Zeit, obgleich er doch auch die harte akademische Wirklichkeit von „Oxbridge“" spiegelt.

Aber zurück zu den Tatsachen, zumindest wie sie Don Martindale referiert: Als junger Mann hatte er vor der Einberufung in das "Pacific Theatre“" des zweiten Weltkriegs Französisch und Deutsch gelernt. Als er 1946 in die USA zurückkommt, um bei Howard P. Becker zu promovieren, stellt er fest, daß seine Sprachfertigkeiten „eingerostet“ sind und beginnt, quasi „zur Übung am soziologischen Hartholz“, Webersche Texte zu übersetzen.

I had, by systematically translating three or four pages a day, gone through "The Religions of India" and was about one quarter of the way through "Ancient Judaism“ when Gerth stopped by the apartment one day on some matter concerning either my dissertation, which was completed and in the hands of the commettee, or house building. We were standing by my bookshelves where the rough translated manuscript was stacked up, when it occurred to me to ask Gerth whether he was willing to check it over for me for accuracy. When I found an opening in his monologue, I took a handful of the manuscript off the top of the pile and asked, „By the way, Gerth, I've been trying to put my German back into working shape, so I have been translating a few pages a day. Would you be willing to check it against the text and give me your impression as to how accurate it is?"

He thumbed through the manuscript. „My God, “ he said "It's Weber. Why are you translating it?"

„If I'am going to practice,“ I explained, „I might just as well do so on things I want to read anyway. I have always wanted to follow up on Weber's general treatment of the hypothesis posed in the ,Protestant Ethic'.“

Gerth flipped through the entire stack of manuscript and discovered that I had gone through the wole of the India volume and had made a fair start on the Judaism volume. „Let's joint translate them,“ Gerth said. „I'll write to Jerry Kaplan of the Free Press tonight. “

Within a week Gerth had obtained joint contracts for our translation of the two volumes. He mentioned incidentally that he intended to do the third book of the trilogy on the 'Religions of China' alone. (Martindale 1982: 113/14)

Ich will auf die weitere Geschichte nicht detailliert eingehen, sondern fasse zusammen: Aus was für Gründen auch immer drängt Gerth seinen Freund, daß zunächst die Studie über das antike Judentum übersetzt werden soll (erscheint 1952; „The Religion of China“ war 1951 erschienen). Nach und nach merkt Martindale, daß gar nicht Hans $H$. Gerth die Übersetzungen überprüft, sondern diese kärrnerische Arbeit an seine Frau delegiert hatte. Als diese dann am 21. Januar 1954 Selbstmord begeht, macht sich Martindale noch einmal selbst an die Arbeit und verfährt wie folgt:

After Gerth's suicide in 1954 it appeared no longer possible, despite interminable tries, to persuade Gerth to get on with the verification of the raw translation of ,The Religion of India'. Finally, in complete despair of ever getting my work out of the project any other way, I went over the raw translation several times myself and put the whole into final shape. I had the typing completed and sent the manuscript to the publisher. It never even occurred to Gerth to offer to share the costs. The volume appeared in 1958, eleven years after the raw translation had been completed. (Martindale 1982: 123)

Das Ergebnis dieses „verzweifelten Versuchs“ ist: „The Religion of India. The Sociology of Hinduism and Buddhism".

War es nur das verlegerische Interesse, das aus "Hinduismus und Buddhismus", dieser kurzen "Zwischenüberschrift" einer Aufsatzsammlung (,Vergleichende religionssoziologische Verche") über "Die Wirtschaftsethik der Weltreligionen" eine „Regionalstudie“, die „Soziologie des Hinduismus und Buddhismus" machte?

Ich glaube nicht, diese Überschrift entspricht vielmehr dem evolutiven Weltverständnis, wie es die amerikanische Nachkriegssoziologie bestimmt hat - und heute noch immer bzw. schon wieder bestimmt. Kommen wir also zum dritten Problembereich.

\section{Die ,parsonifizierte“ Vermittlung - Weber als Entwicklungstheoretiker}

Schon die kurze editorische Fußnote zur Paperback-Ausgabe (1967) des Werkes ist bezeichnend, wenn es heißt:

The central concern of this and others of Weber's studies of countries we today describe as „developing“ was with the obstacles to industrialization and modernization. Weber anticipated by several decades a problem that has come to occupy the post-World War II world. Why had these countries failed to display the full consequences of those rationalizing tendencies which, to Weber's mind, had so powerful an affinity with the scientific-technical transformation of the West?

Mit dieser Einleitung wurde Max Webers Studie dem naiven Modernisierungsverständnis der ersten Entwicklungsdekade zugeordnet, als man noch an Fortschritt als einen sich selbst rechtfertigenden Prozeß zunehmender Industriealisierung glaubte und der festen Überzeugung war, er sei mit wachsender Güterproduktion, steigendem Energieverbrauch, hohem Zeitungsausstoß pro Kopf der Be- 
völkerung, Urbanisierung, mindestens einem Kraftwagen pro Kernfamilie identisch und über entsprechende Indikatoren auch interkulturell gültig ablesbar.

So kann man sich auch auf einem von dem Ehepaar Loomis betreuten und von der Ford Foundation finanzierten Weber-Seminar im National Institute of Community Development (Hyderabad) 1966 fragen:

What one would like to know is this: Under what conditions, if any, can India hope to become an industrial nation? Does such a process require a radical break with the past? How is such a break possible? What would be the foreseeable consequences? Does a radical break with the past, if at all necessary, require a Communist solution? Or, are there aspects of the Indian tradition which are favourable to industrial development? (Loomis/Loomis 1969: v)

Damit beginnt in Indien die Suche nach den ,functional equivalents" für den Modernisierungsprozeß, eine Suche, die selbst vor Gandhi nicht haltmacht und ihn identifiziert als einen , hard working ascetic“, der angeblich genau „the Weberian notion of the ascetic Protestant" entsprach. (Loomis/ Loomis $1969: 20$ ). Gründlicher kann man eigentlich weder Gandhi noch Weber mißverstehen!

Ich hatte in meiner Einleitung gesagt, daß Weber „parsonifiziert" vermittelt wurde, indem man seine Kontrastanalyse zur spezifisch abendländischen Entwicklung einordnete in das evolutionäre Schema, wie es von der Parsonschen Schule mit immer weiterreichenden Wirkungen vorgetragen wurde. (Parsons: 1966)

Wie stark Talcott Parsons bei seiner Übersetzung der „Vorbemerkung“ und der Arbeit über die protestantische Ethik in Kategorien von „development" dachte, habe ich an zwei zentralen Beispielen an anderer Stelle anzudeuten versucht (Kantowsky 1982a: Fn. 14). Dem sei jetzt noch ein „Bekenntnis“ von Parsons aus einem Brief zugefügt, in dem er zwei Monate vor seinem Tod (auch er starb in München!) an Edward A. Tiryakian in Reaktion auf ein Manuskript über die Herkunftsgeschichte der Metapher vom ,iron cage“ schrieb:

it seems to me that the Weber of the „Author's Introduction" fits your interpretation of having mitigated the pessimism about the iron cage which he expressed in the closing paragraphs of ,The Protestant Ethic ${ }^{*}$. . . All of this is of course in close accord with my own development as a student and interpreter of Weber's work. I have been moving toward a substantially more optimistic, if you will, interpretation of modern industrial society than is the predominant vogue among sociologists and related types of intellectuals. At the same time, I have maintained my basic confidence in Weber's work and the extraordinary levels of insight he attained. (Parsons 1981: 35/36)

\section{Dazu folgende Bemerkungen:}

- Weber benutzte das Gleichnis ,stahlhartes Gehäuse" (GAzRS I, 203) in einer langen Passage, die schon 1904/05 in der ersten Fassung der Arbeit im "Archiv" zu finden ist, um seine pessimistische Einschätzung des kapitalistischen Entwicklungsweges $\mathrm{zu}$ illustrieren. Es ist aber mitnichten so, daß Weber dann später bei seinen Reflexionen über gegenwärtige Trends und Strukturen das Bild nicht mehr verwendet und seinen Pessimismus abgemildert hätte. Im Gegenteil, er verwendet die Phrase immer dann, wenn er sich prophetisch-düster äußert:

- „Zur Lage der bürgerlichen Demokratie in Rußland“, erschienen 1905/06 (Weber 1980, 63).

- Über „Wahlrecht und Demokratie in Deutschland", erschienen im Dezember 1917 (Weber 1980, 254).

- Über „Parlament und Regierung im neugeordneten Deutschland", erschienen im Sommer 1918 (Weber 1980, 331f.).

Dieser skeptische späte Weber, der an der Zukunft des Sonderweges abendländischer Entwicklung immer stärker leidet, wird bei der Rückvermittlung seiner Studien nach Osten (und nicht nur nach Indien) systematisch unterschlagen (vgl. Weyer 1984). Er muß wieder (nicht nur in Indien) freigelegt werden von einer neuen Generation von Sozialwissenschaftlern, die, wie Weber und mit seinen Instrumenten, erkannt haben, daß das „Getriebe jenes liebeleeren und erbarmungsfremden ökonomischen Kampfs ums Dasein, den die bürgerliche Phraseologie als ,friedliche Kulturarbeit" bezeichnet: eine andere Form des Kampfes des Menschen mit dem Menschen, bei der nicht Millionen, sondern hunderte von Millionen jahraus, jahrein an Leib und Seele verkümmern" (Weber 1916, siehe: Weber 1980: 144) uns „mit überwältigendem Zwange bestimmt und vielleicht bestimmen wird, bis der letzte Zentner fossilen Brennstoffs verglüht ist“" (Weber 1904/05, siehe GAzRS I, 203).

$\mathrm{DaB}$ wir uns das Ende dieses Kampfes aber nicht nur mehr als ein "final whisper“, sondern inzwischen auch als "great bang“" vorstellen und den Exterminismus als letztes Stadium der Zivilisation nach Megatonnen hochrechnen können (Thompson 1980), darin liegt die Fortschrittlichkeit einer spezifisch abendländischen Form der Weltbearbeitung und -beherrschung. Fragen wir uns daher zum 
Schluß noch, woran es wohl liegen mag, daß sie dennoch von der sozialwissenschaftlichen Intelligenz Indiens weithin angenommen wurde? Warum übernahm sie die Fremddeutungen der eigenen Gesellschaft als defizitär und modernisierungsbedürftig? Litt sie denn etwa an den Zuständen der eigenen Gesellschaft?

Ganz im Gegenteil - wer den Lebensstil indischer Kollegen kennt, der wird sich schon so manches Mal gefragt haben, was es denn eigentlich ist, das sie so vehement für das "stahlharte Gehäuse“ optieren läßt. Wieso verwerfen sie die hinduistische Theodizee des Leids, den einen der drei in sich geschlossenen Daseinsentwürfe, die menschliche „Weltdeutung“ nach Weber insgesamt nur hervorzubringen vermochte und versuchen stattdessen eine Theodizee des Glücks zu entwickeln?

\section{Das Glück will legitim sein}

Webers Ausführungen über die Brahmo Samaj paraphrasierend, muß man sagen, daß wir es bei der indischen sozialwissenschaftlichen Intelligenz mit einer bürgerlichen Intellektuellenschicht $\mathrm{zu}$ tun haben, deren Interessen auf das engste verwoben sind mit den Maßnahmen einer Politik zur Durchsetzung des westlichen Paradigmas von Modernisierung und Entwicklung (GAzRS II, 362). Es schallt also aus ihrem Munde nur das zurück, was wir über die vielfältigen Instrumentarien der Bildungs- und Entwicklungshilfe in ihre Köpfe einzupflanzen versucht haben.

Vor allem aber gilt auch für sie die folgende allgemeine Feststellung Max Webers aus der „Einleitung“: „Der Glückliche begnügt sich selten mit der Tatsache des Besitzes seines Glückes. Er hat darüber hinaus das Bedürfnis: auch noch ein Recht darauf zu haben. Er will überzeugt sein, daß er es auch ,verdiene'; vor allem: im Vergleich mit andern verdiene. Und er will also auch glauben dürfen, daß dem minder Glücklichen durch den Nichtbesitz des gleichen Glückes ebenfalls nur geschehe, was ihm zukommt. Das Glück will ,legitim* sein." (GAzRS I, 242).

Nicht also als Kritiker, sondern nur als Apologet einer bestimmten, angeblich universellen und durch die „New I.ead Societies“ vorgezeichneten Entwicklungsrichtung konnte Weber in Südasien rezipiert werden. Webers historische Kontrastanalyse von „Hinduismus und Buddhismus“ mußte von dieser Schicht als lïcken- und fehlerhafte Darstellung der eigenen Gegenwart verworfen wer- den, um die generelle Modernisierungsfähigkeit des Landes behaupten sowie Schlüsselrollen bei der entsprechenden Neustrukturierung von Erziehung und Verwaltung reklamieren zu können. Anstatt, bei Weber ansetzend, weiterzufragen, in welcher Richtung denn (- wenn schon der Geist des Kapitalismus aus der Mitte der durch spezifisch andersartige Mächte beherrschten südasiatischen Gemeinschaften nicht hatte geboren werden können -; vgl. GAzRS, II, S. 359) eine Entwicklungschance für Indien (- trotz des artefaktischen Imports von kapitalistischen Strukturen durch das internationale System -; vgl. GAzRS, II, S. 359) heute möglich wäre, bemühen sich die indischen Sozialwissenschaftler, die skeptischen Einschätzungen (,wie wenigstens wir uns gern vorstellen“; Einschub im ersten Satz der ,Vorbemerkung zu GAzRS, I) des späten Weber zu bestätigen, daß der auf dem Boden des Okzidents aufgetretene Rationalismus der Weltbeherrschung doch eine Entwicklungsrichtung von universeller Gültigkeit vorgezeichnet habe.

Wer die destruktive Expansion der abendländischen Lebensform mit Entwicklungstheorien und -modellen als unausweichliche evolutive Logik untermauern will, der mag das tun. Doch Max Weber und seine Gesammelten Aufsätze zur Religionssoziologie lasse man dabei aus dem Spiel.

Da ich mich nicht für das halte, was man einen Max Weber Kenner nennt, erlaube ich mir, meine Ausführungen mit einigen bestätigenden Passagen aus einem längeren Brief zu ergänzen, den mir der Altmeister der Weber-Interpreten, Johannes Winckelmann, am 25. September 1980 in dieser Angelegenheit geschrieben hat:

Die „übergeordnete Fragestellung“ allgemeinster Art der drei eine Einheit bildenden vergleichenden Studien - zu denen ausweislich der Verlagsunterlagen noch ein vierter Band hinzukommen sollte, der den Anschluß an die vorreformatorische Periode herzustellen hatte, ist die nach den „universalgeschichtlichen Zusammenhänge von Religion und Gesellschaft" (GAzRS I, S. 206 Anm. 1 a. E.). Innerhalb dieses Rahmens ist die Unterfragestellung der vergleichenden Untersuchung auf ihrem Wege ,von Ost nach West" (GAzRS I, S. 267 Anm. 1) nicht die Kulturanalyse noch so gedrängter Natur der behandelten asiatischen und vorderorientalischen Regionen als solcher, sondern vielmehr die Gewinnung von „Vergleichspunkten" (sic! s. GAzRS I, S. 12f., 15), um vor diesem gedanklichen Hintergrund als Kontrastentwicklung (GAzRS I, S. 13 o.) die besondere Eigenart und Wirkung der ,okzidentalen Kulturreligionen“ (a.a.O.S. 15) aufzudecken und überhaupt die Spezifika der unwiederholten europäischen Kulturwelt (a.a.O. S. 1) deutlich hervortreten zu lassen und tunlichst eine Antwort auf die Frage zu 
gewinnen: wieso gab es die spezifisch abendländischen Kulturerscheinungen nur hier? (GAzRS I, S. 1).

Niemals ist Max Weber auf die Idee verfallen, den östlichen Völkern „den Spiegel vorhalten“, sie etwas „belehren" oder gar zur Übernahme der europäisch-amerikanischen Zivilisation der rationalen Weltbeherrschung bereden zu wollen. Seine Soziologie war ausdrücklich ihrer Grundmaxime nach ,neutral'. Etwas derartiges kam ihm also zu keiner Zeit in den Sinn, umso weniger als er selbst der Auffassung war, bereits Anzeichen von „Tendenzen zur inneren Umbildung des Kapitalismus" wahrnehmen zu können (Schluß seines Programms für den GdS). In dieser einsetzenden Dämmerung hatte für ihn die Eule der Minerva ihren abendlichen Flug bereits begonnen.

\section{Zwei Schlußbemerkungen}

1. „Geht nicht nach Hörensagen, Kalamer, nicht nach Überlieferungen, nicht nach Tagesmeinungen, nicht nach der Autorität heiliger Schriften, nicht nach erdachten Theorien und bevorzugten Meinungen." Dies war die Antwort des Asketen Gotama auf die Frage der Kalamer, was sie denn machen sollten, ihnen würde von durchziehenden Lehrern mal das eine und dann wieder das völlig andere verkün$\operatorname{det}$ (Die Lehrreden Bd. I: 168).

Also: „Kommt her und seht selbst.“ Die Weberschen Texte machen einem das zwar nicht immer ganz einfach, dennoch sind sie es wert, daß man sie im Original liest und sich nicht „beraten“ läßt. Dazu noch einmal Johannes Winckelmann in dem oben schon zitierten Brief vom 25. September:

Man muß sich darüber im klaren sein, daß Max Weber in den Vereinigten Staaten immer nur schubweise und partiell und immer nur im Kontext der inneramerikanischen Diskussionslage rezipiert worden ist: das eine Mal gegen den Pragmatismus und Behaviorismus, das andere Mal für den strukturellen Funktionalismus und symbolic interactionalism. Dies sind nur wenige Beispiele. Das erste Mal, daß ein Werk Max Webers in toto, d. h. zugleich mit seinem eigenen Verständnishorizont, in amerikanischer Übersetzung in Erscheinung trat, geschah, als Günter Roth und Claus Wittich ,Economy and Society" als geschlossene Werkseinheit dem amerikanischen Lesepublikum präsentierten (1968) und in seinen inneren Zusammenhängen und mit seinen gedanklichen Voraussetzungen kommentierten.

Hoffen wir also, daß die in Arbeit befindliche „Max-Weber-Gesamtausgabe“ (MWG) es bald vielen ermöglichen wird, Max Webers Gedankengänge in all ihrer historischen Bedingtheit zu verfolgen. Dazu teilte mir allerdings Horst Baier, einer der fünf Herausgeber im Dezember $1983 \mathrm{mit}$, daß unsere Generation wohl mehr als fünfzehn der auf insgesamt über fünfundzwanzig Bände zu veranschlagenden MWG nicht erleben werde.

Diejenigen also, die auch weiterhin keinen $\mathrm{Zu}$ gang zu den unveröffentlichten Materialien und besonders den Briefen Max Webers haben und das sind auf Grund interner Absprachen außer dem kleinen Kreis der Herausgeber und für einzelne Bände verantwortlichen Editoren leider alle Wissenschaftler der Gegenwart! -, werden noch für geraume Zeit auf das angewiesen sein, was sich aus vorliegenden Texten durch Vergleiche mit früheren Fassungen und sorgfältige Lektüre der Fußnoten erschließen läßt. Es ist ein bescheidener Trost, daß auch die Herausgeber der MWG unter der Geheimhalte-Klausel, auf die sie sich intern verpflichtet haben, zu leiden beginnen. So verdienstvoll Wolfgang Schluchters neuerliche Auseinandersetzung mit den Argumenten Friedrich Tenbrucks auch sein mag (Schluchter 1984), ein öffentlicher Vorgriff auf die Herausgabe der Korrespondenz Webers hätte seine ,werksgeschichtliche Rekonstruktion" sicher eindeutiger und kürzer (vielleicht ja aber auch anders?) ausfallen lassen. Abzuwarten bleibt, wie Horst Baier mit diesem Problem anläßlich der Fertigstellung seiner schon mehrfach angekündigten Max-Weber-Biographie umgehen wird.

2. „Den Dingen geht der Geist voran, der Geist entscheidet" heißt es im „Dhammapada“, dem „Wahrheitspfad“ des Buddhismus. Max Weber formuliert in der zweiten Fassung der „Einleitung" weniger idealistisch, daß die Weltbilder, welche durch Ideen geschaffen werden, quasi als Weichensteller die Bahnen bestimmten, ,in denen die Dynamik der Interessen das Handeln fortbewegte" (GAzRS I: 252).

Insofern sind Versuche, einen entamerikanisierten Weber nach Indien zu vermitteln (Kantowsky 1984a) vielleicht doch nicht ganz irrelevant. Vor allem aber habe ich bei der Anregung und Gestaltung von Max-Weber-Konferenzen in New-Delhi (1.-3. März 1984) und Colombo (12.-14. März 1984) versucht (Kantowsky 1984b), als Sozialwissenschaftler der Handlungsanweisung Max Webers gerecht zu werden, d. h. dem Dämon gehorcht (vgl. Weber $1922:$ 555), der meines Lebens Fäden hält (Kantowsky 1985). 


\section{Literatur}

Die Lehrreden des Buddha aus der Angereihten Sammlung. 1969. Neue Gesamtausgabe in fünf Bänden. Aus dem Pali übersetzt von Nyanatiloka. Köln: DuMont Schauberg.

Ernst, W./Kantowsky, D. 1983. Mad Tales from the Raj: Case Studies from Pembroke House and Ealing Lunatic Asylum, 1818-1892. Paper pres. to „Eighth European Conference on Modern South Asian Studies". Tällberg (Schweden), 2. - 8. 7. 1983. Gekürzt in: Society, Vol. 22, No. 3 (1985), 31-38.

Gerth, H. H. and C. W. Mills. 1948. From Max Weber: Essays in Sociology. Translated, edited and with an introduction of $\mathbf{H}$. H. Gerth and C. Wright Mills. London: Routledge and Kegan Ltd.

Kantowsky, D. 1981. Max Weber on India and Indian Interpretations of Weber. Paper presented to the 7th European Conference on Modern South Asian Studies. London, School of Oriental and African Studies, 7-11 July, 1981. Jetzt veröffentlicht in: Changing South Asia: Religion and Society. Edt. by K. Ballhatchet \& D. Taylor. Hong Kong: Asian Research Service, 1984 11-35.

Kantowsky, D. 1982a. Die Rezeption der Hinduismus/ Buddhismus-Studie Max Webers in Südasien: ein Mißverständnis? Arch. europ. sociol. XXIII: 317-355.

Kantowsky, D. 1982b. Max Weber on India and Indian interpretations of Weber. Contributions to Indian Sociology (NS), Vol. 16, No. 2: 141-174.

Kantowsky, D. 1984a. Max Weber's Contributions to Indian Sociology. In: Contributions to Indian Sociology (NS), Vol. 18, No. 2, 307-317.

Kantowsky, D. 1984b. Max Weber in Südasien - Bericht über zwei Konferenzen in New Delhi und Colombo im März 1984. In: Internationales Asienforum, Vol. 15, No. 2. S. 348-351.

Kantowsky, D. 1985. Von Südasien lernen. Erfahrungen in Indien und Sri Lanka. Frankfurt/New York: Edition Qumran im Campus Verlag.
Loomis, Ch. P. and Z. K. Loomis, eds. 1969. Socioeconomic change and the religious factor in India. An Indian symposium of views on Max Weber. New Delhi: Affiliated East-West Press.

Martindale, D., 1982. The Monologue: Hans H. Gerth (1908-1978), A Memoir. Ghaziabad (India): Intercontinental Press.

Parsons, T. 1966. Societies: evolutionary and comparative perspectives. Englewood Cliffs, N. J.: Prentice Hall.

Parsons, T. 1981. Excerpts from a letter to Tiryakian. Sociological Inquiry, Vol. 51, 35-36.

Schluchter, W. 1984. Max Webers Religionssoziologie. Eine werkgeschichtliche Rekonstruktion. In: KZfSS, Vol. 36, 342-365.

Thompson, E. 1980. Notes on exterminism, the last stage of civilization. New Left Review 121: 3-31.

Weber, M. 1921. Hinduismus und Buddhismus. Gesammelte Aufsätze zur Religionssoziologie, Bd. II. Tübingen: J. C. B. Mohr (Paul Siebeck).

Weber, M. 1922. Gesammelte Aufsätze zur Wissenschaftslehre. Tübingen: J. C. B. Mohr (Paul Siebeck).

Weber, M. 1930. The protestant ethic and the spirit of capitalism. Translated by Talcott Parsons. With a Foreword by R. H. Tawney. London: Allen \& Unwin.

Weber, M. 1958. The religion of India. The sociology of Hinduism and Buddhism. Transl. and edited by Hans H. Gerth and Don Martindale. Glencoe: The Free Press.

Weber, M. 1980. Gesammelte politische Schriften. Tübingen: J. C. B. Mohr (Paul Siebeck), 4. Aufl.

Weber, M. 1982. Sociologica della Religione. 2 Bde. Milano: Edizioni di Communità.

Weyer, J. 1984. Westdeutsche Soziologie 1945-1960. Deutsche Kontinuitäten und nordamerikanischer Einfluß. Berlin/München: Duncker \& Humblot. 